\title{
Carnets
}

Revue électronique d'études françaises de l'APEF

Première Série - 4 | 2012

(Res)sources de l'extravagance

\section{Théâtre(s) sous un crâne}

Mallarmé et Pessoa (d'Igitur au Faust Tragédie subjective)

Maria de Jesus Cabral

\section{(2) OpenEdition}

Journals

Édition électronique

URL : http://journals.openedition.org/carnets/6975

DOI : $10.4000 /$ carnets.6975

ISSN : 1646-7698

Éditeur

APEF

Édition imprimée

Date de publication : 1 janvier 2012

\section{Référence électronique}

Maria de Jesus Cabral, « Théâtre(s) sous un crâne », Carnets [En ligne], Première Série - 4 | 2012, mis en ligne le 31 janvier 2012, consulté le 01 mai 2019. URL : http://journals.openedition.org/ carnets/6975; DOI : 10.4000/carnets.6975

\section{(c) (7) (9)}

Carnets est mis à disposition selon les termes de la licence Creative Commons - Atribution - Pas d'utilisation commerciale 4.0 International. 


\title{
THEATRE(S) SOUS UN CRANE \\ Mallarmé et Pessoa (d'Igitur au Faust Tragédie subjective) ${ }^{1}$
}

\author{
MARIA DE Jesus REIS CABRAL \\ Centre de Littérature Portugaise \\ Université de Coimbra \\ mariajesus@fl.uc.pt
}

\begin{abstract}
Résumé
La présente étude se propose d'interroger la notion de théâtre mental, capitale dans la pensée et dans l'œuvre de Stéphane Mallarmé, tachant de l'élucider dans les rapports suscités avec la conception dramaturgique 'extra-vagante' déployée par Fernando Pessoa notamment dans le processus de création hétéronymique. Son ex-centricité est envisagée par la prise en compte de l'élément psycho-pathologique et au moyen des concepts critiques de manière et de folie établis par G. Dessons $(2004,2010)$. Rapprocher Igitur ou la Folie d'Elbehnon, et Faust, tous deux inachevés dans un éclectisme générique déjouant toute positivité est l'objet du troisième volet de notre lecture.
\end{abstract}

\section{Abstract}

The stepping stone of this paper is the explanation of the concept of mental theatre, core to Stéphane Mallarmé's thought and work, in order to assess the links it establishes with the theatrical concept of 'extra-vagant', by Fernando Pessoa, namely in his process of heteronym creation. Its ex-centricity shall be equated based on the psycho-pathological element and through the critical concepts of manner and madness as laid down by Gérard Dessons $(2004,2010)$. The The third part of this paper deals with a comparative approach to Igitur or la Folie d'Elbehnon and Faust, tragédie subjective, both left unfinished and both part of an eclectic gender against any form of positivity.

Mots-clés: Mallarmé, Pessoa, théâtre mental, hétéronymie, manière

Keywords: Mallarmé, Pessoa, mental theatre, heteronymism manner

\footnotetext{
${ }^{1}$ Cet article a été élaboré dans le cadre du projet "Rumos do teatro poético, dos impulsos novistas finisseculares às viragens de Orpheu", subventionné par la Fundação para a Ciência e a Tecnologia et intégré dans le Programme européen POPH/FSE.
} 
"Au centre de la dite circonférence/ je le voyais en-dedans, en perspective infinie"

(Fernando Pessoa, Faust, tragédie subjective ${ }^{2}$ )

“... Je me souvins d'Averroës qui, prisonnier de la culture de l'Islam, ne put jamais savoir la signification des mots tragédie et comédie. Je racontai son aventure; à mesure que j'avançai, j'éprouvai ce que dut ressentir ce dieu mentionné par Burton qui voulut créer un taureau et créa un buffle. Je compris que mon œuvre se moquait de moi. Je compris qu'Averroës s'efforçant de s'imaginer ce qu'est un drame, sans soupçonner ce qu'est un théâtre, n'était pas plus absurde que moi /.../ que mon récit était un symbole de l'homme que je fus pendant que je l'écrivais et que, pour rédiger ce conte, je devais devenir cet homme et que, pour devenir cet homme, je devais écrire ce conte, et ainsi de suite à l'infini" (Borges, 1967: 129-130).

Le vertige de l'infinité comme parabole de la création littéraire, susceptible de prendre des formes hybrides, voire nouvelles, et déjouant la fixité du centre au profit du mouvement circulaire, allégrement contradictoire, s'offre comme entrée dans cette lecture comparatiste de Mallarmé et Pessoa sous le signe d'une dramaturgie extravagante - au sens suscité par l'étymologie composite du mot: extra - du latin "en-dehors de" et vagans, du latin vagari "errer" -, soit une dramaturgie pensée et conçue hors des limites ou contraintes ${ }^{3}$ et inventant, par tentatives - par erreurs et errances - de nouveaux rapports entre l'homme et le monde.

Sans trop m'y attarder, je commencerai par esquisser les traits essentiels du théâtre mental de Mallarmé et certains liens avec la conception dramatique de Pessoa, notamment la question de l'hétéronymie, qui sera examinée par l'attention à l'élément psychopathologique et au moyen du concept de manière établi par G. Dessons (2004, 2010). Rapprocher Igitur ou la Folie d'Elbehnon, daté de 1869-70 et publié une cinquantaine d'années plus tard ${ }^{4}$, et Faust, composé entre 1908 et 1933 et édité sous la forme de "Tragédie subjective" en 1985, tous deux inachevés et générant un éclectisme singulier, constitue la troisième étape de cette étude.

\footnotetext{
${ }^{2}$ Traduit du portugais par Pierre Léglise-Costa et André Velter (Pessoa, 2008: 52). Toutes les citations du Faust renvoient à cette édition.

${ }^{3}$ II est à cet égard pertinent de rappeler un des exemples donnés par le Dictionnaire Latin-Français de Gaffiot, qui reprend une citation du De Oratore de Cicéron: "Ne pas être soumis à des contraintes de rythme" $(3,176)$.

${ }^{4}$ Dont le manuscrit ne fut publié qu'en 1925 par Edmond Bonniot, gendre de Mallarmé. Cf. la "Notice" d'Igitur établie par Bertrand Marchal (Mallarmé, 1998: 1346-1353).
} 


\section{“...théâtre de notre esprit, prototype du reste” (Mallarmé, Hamlet)}

La recherche d'un théâtre scandé par le mystère, et désavouant le principe de relation entre la forme et le contenu - et autres dualismes de tradition aristotélicienne - est un dénominateur commun à Mallarmé et à Pessoa; ce projet qu'ils n'ont pas réalisé de leur vivant se trouve à la source des expériences dramaturgiques les plus avant-gardistes, jusqu'à l'actualité. Par-delà les catégories et les procédés canoniques, leur théâtre consacre la prévalence de l'intérieur. L'idée d'une tragédie autre qu'une "imitation de la vie confuse et vaste" (Mallarmé, 2003: 294), s'impose en effet tôt à Mallarmé et l'oriente dès 1865 vers une "poétique de l'effet" et une nouvelle Hérodiade, "non plus tragédie, mais poème" (Mallarmé, 1995: 253) au moment où le Faune, "impossible au théâtre mais exigeant le théâtre" (ibid.), était refusé par Banville et Coquelin à la Comédie Française. Et le mot mystère, qui s'offrait déjà plein de promesses trois années auparavant au jeune poète d'Hérésies Artistiques, est pour le poète-dramaturge travaillant à la "Scène d'Hérodiade" le plus approprié à la représentation d' "un être purement rêvé et absolument indépendant de l'histoire" (ibid.).

En 1893, dans "Planches et Feuillets", Mallarmé frayait ce chemin vers le "drame moderne' (Szondi, 1950) à contre-courant du rationalisme cartésien: "le théâtre, inhérent à l'esprit, quiconque d'un œil certain regarda la nature, le porte avec soi, résumé de types et d'accords" (Mallarmé, 2003: 195, je souligne). Le théâtre est pensé non plus comme représentation mais comme dispositif inscrit dans le sujet lui-même et capable d'ouvrir des territoires de création multiples et, geste de premier ordre, de saisir la corrélation entre l'homme et le monde. Un "Livre, explication de l'homme" (Mallarmé, 1998: 786): c'est tout le sens (direction et signification) de l'entreprise mallarméenne.

Le théâtre re-noue avec l'homme, dont la "nature" est merveilleusement fabuleuse, comme l'avait suggéré Descartes dans ses Méditations philosophiques. À ce stade, le théâtre transporte la complexité de la personne humaine, ses connexions intimes à la pensée, à la parole et au monde. Esquissant, dans "Le genre ou des Modernes" une "théorie tragique actuelle", Mallarmé propose un théâtre hors catégories, organiquement solidaire de la vie intérieure: "le drame, latent, ne se manifeste que par une déchirure affirmant l'irréductibilité de nos instincts" (Mallarmé, 2003: 188).

Tout un tournant, y compris épistémologique, s'énonce et s'annonce là, la question du théâtre n'étant plus octroyée par une structure ou par un mécanisme (externe): c'est une extension du vivant au point de rencontre entre le sensible et l'intelligible, l'intérieur et l'extérieur, propice donc à des réalisations variées et multiples. Un positionnement avantageux pour l'homme qui le place en deçà (et au-delà) d'une obédience strictement rationnelle au profit d'une latence - mot très mallarméen - foncièrement poétique. L'homme engendre, pour ainsi dire, le théâtre et ce n'est pas le théâtre qui (re)produit l'homme. Aussi 
Mallarmé célèbre-t-il à propos de Hamlet le prestige de ce "personnage unique d'une tragédie intime" (Mallarmé, 2003:166), en action même dans son intériorité. Le doute a frappé de son "jeu suprême", le procès de la grammaire classique - engagé, nous l'avons dit, en amont par Victor Hugo ${ }^{5}$ - et opéré de manière radicale par les "extravagants du théâtre" ${ }^{\text {"6 }}$ du premier XXème siècle. Artaud entre tous prône une écriture qui "s'éclaire de l'intérieur" (Artaud, 2004:147) sans dissocier chair et connaissance, nerfs et mots; une écriture issue de "la finesse des moelles", comme il l'écrit dans "Position de Chair" (1925)

En filigrane, l'image célèbre de Hamlet contemplant le crâne de Yorick, rappelle la dette de l'auteur d'Hernani envers l'auteur élisabéthain ${ }^{8}$ et, symboliquement, de toute une théâtralité 'moderne' largement redevable de performances spectrales ${ }^{9}$. Et si le titre de tel chapitre des Misérables - "La tempête sous le crâne" - peut nous transporter au cœur du théâtre shakespearien et à la scène du naufrage de La Tempête, l'on ne saurait occulter l'attachement réel du poète du Coup de dés comme de celui de l' "Ode Maritime" à l'égard du grand dramaturge.

C'est en effet en rendant hommage à Shakespeare que Pessoa célèbre à son tour le "monologue prolongé et analytique" (Pessoa, 1966:106) comme l'armature de son art dramatique. Le monologue, qui détient un rôle primordial dans Hérodiade, dans le Faune, et dans Igitur, est aussi la forme privilégiée par Pessoa. II y loge notamment son drame poétique Le Marin où, pour reprendre les mots de Teresa Rita Lopes, les Veilleuses "ne constituent qu'un seul monologue à trois voix" (Lopes, 1985: 195). Contrairement au dialogue, toute la tension dramatique se joue dans la déambulation intérieure de ces personnages, au carrefour de leurs voix intérieures, qui figurent plus que tout la prégnance de la mort dans une pièce dégagée de l'anecdote. Comme nous le verrons, le monologue est le nœud du conflit intime du Faust dérogeant le dialogue et l'action pour révéler ses avers intérieurs, le monde caverneux de l'esprit, sorte de théâtre sur lequel se succèdent plusieurs plans de 'visions' en infinie circularité...

\footnotetext{
${ }^{5}$ Inapproprié à rendre compte de l'hétérogénéité de l'homme et de la nature. Dans sa "Préface de Cromwell" (1827) Hugo posait notamment le mélange de "tous les contraires" comme un des ressorts du drame nouveau et hissait le grotesque au sommet des valeurs poétiques: “... le drame c'est le grotesque avec le sublime, l'âme sous le corps, c'est une tragédie sous une comédie" (Hugo, s/d: 67).

${ }^{6}$ Voir à ce sujet le catalogue de l'exposition homonyme, présenté par Geneviève Latour (Latour, 2000), où figurent les noms bien connus de Jarry, Apollinaire, Cocteau, Radiguet, Satie, Max Jacob, Marinetti, Ghelderode, Artaud, Vitrac, Barrault, Prévert et Picasso, entre autres.

${ }^{7}$ Une autre ligne de rapprochement se profile ici, qui dépasse toutefois le cadre de cette contribution: l'écriture elle-même conçue comme spectacle du vivant, imprimée des vibrations de l'esprit sur la chair, qu'il tiendrait au texte de corporiser. Et au lecteur d'inspecter (in-spectare), de découvrir de l'intérieur.

8 "Shakespeare, c'est le Drame; et le drame, qui fond sous un même souffle le grotesque et le sublime, le terrible et le bouffon, la tragédie et la comédie" écrit Victor Hugo dans la "Préface" de Cromwell (Hugo, s/d: 28).

${ }^{9} \mathrm{Je}$ songe notamment aux créations fantasmagoriques à la limite du théâtre de Denis Marleau (de la compagnie canadienne UBU), tirant parti des jeux d'intermédialité pour placer le texte littéraire en premier plan, l'exhiber dans son essence (sens, rythme, voix), dans sa spectralité. Je me permets de renvoyer à mon article "En spectacle (in)interrompu: Pessoa, Maeterlinck, Beckett selon Denis Marleau" (Cabral 2012, à paraître).
} 
Mais le motif du crâne voisine aussi avec celui de cerveau et convient à deux poètespenseurs chez qui l'energeia créatrice relève du labeur autant que de la spéculation poétique. Mallarmé 'algébriste du langage', selon le mot de Valéry, assemblant des "entrelacs distants" des fragments d'Hérodiade, du Faune ou d'Igitur aux filaments du Coup de dés - comme autant de "divisions prismatiques de l'Idée" (Mallarmé, 2003: 391). L'œuvre mallarméenne est en effet ce lieu "architectural et prémédité" où tout est interrelié en (é)toile d'araignée. Rien de mieux pour égarer le lecteur maladroit que ce jeu mental et dynamique "identifiant la scène et la salle".

\section{“Dubius adfectibus errat” (Ovide, Métamorphoses)}

Proche de Mallarmé par une conception poétique comme énigme et 'action restreinte', qui ne saurait être déliée de la lucidité d'une pensée, autre est le jeu de Fernando Pessoa, et bien plus extravagantes les manières de le livrer. Entrer dans l'univers du mentor intellectuel d'Orpheu qui se disait "forme synthèse de tous les mouvements littéraires modernes", c'est quitter, pour les déborder, les champs établis pour valider également l'incertain et l'errance. Pour entrer dans le paradoxe, qui sert d'humus à toute son œuvre. Et le cerveau par lequel passent "différents acteurs, jouant différentes pièces" en constitue le noeud, lieu et lien de relation entre le monde connu et ses périphéries en libre devenir.

L'hétéronymie pessoenne est comme l'aleph du bibliothécaire de Babel, ce jeu de points de vues se démultipliant à partir d'un même centre et s'inscrit, au fond, dans la continuité de la dramaturgie mentale mallarméenne, pensée en dehors de la représentation, pensée à l'intérieur du sujet - dans cette "scène de théâtre" que constitue le cerveau de Pessoa, selon sa propre évocation. La diffraction poétique en hétéronymes, soushétéronymes ou semi-hétéronymes, qui se croisent et se superposent à sa propre figure telle le brossent de nombreux portraits - montre qu'il existe des connexions interactives et artistiquement déterminantes entre corps et langage, entre raison et sensation, entre sujet et drame. Nul n'a mieux décrit le fondement critique et la condition éminemment théâtrale d'une telle dépersonnalisation que l'écrivain lui-même. Je rappelle un passage d'une de ses fameuses lettres à Gaspar Simões, celle du 11 décembre 1931:

Le point central de ma personnalité, en tant qu'artiste, c'est que je suis un poète dramatique; j'ai sans cesse, dans tout ce que j'écris, l'exaltation intime du poète et la dépersonnalisation du dramaturge. Je m'envole autre - c'est tout. Du point de vue humain - auquel le critique n'a pas à toucher, car cela ne lui sert à rien - je suis un hystéroneurasthénique, l'élément hystérique prédominant dans l'émotion, l'élément 
neurasthénique dans le domaine de l'intelligence et de la volonté (méticulosité de l'une, faiblesse de l'autre) (Blanco (org.), 1986: 279).

Le trait clinique - "hysteroneurasthénique" - de Pessoa est inséparable de cette dynamique de création - paradoxale et potentiellement nihiliste -, d'une "coterie inexistante", qu'il fait varier dans des registres multiples, mais toujours reliés à lui-même par un rapport dialogique ${ }^{10}$. Le poète récidive en critique, évoquant la nuit convulsive de la genèse de ses hétéronymes ${ }^{11}$ :

Ce fut le 8 mars 1914 - je m'approchai d'une commode haute, et prenant un papier, je commençai d'écrire, debout, comme je le fais chaque fois que je le peux. Et j'écrivis trente et quelques poèmes d'affilée, dans une sorte d'extase dont je ne parviendrai à définir la nature. Ce fut le jour triomphal de ma vie et je n'en retrouverai jamais d'autre semblable. Je débutai par un titre: "Le Gardeur de troupeaux" et ce qui suivit fut l'apparition en moi de quelqu'un à qui je donnai aussitôt le nom d'Alberto Caeiro. Pardonnez-moi l'absurde de la phrase: mon maître m'était apparu. Telle fut la sensation immédiate que j'eus. A tel point que, une fois écrits ces trente et quelques poèmes, je m'emparai aussitôt d'un autre papier sur lequel j'écrivis, d'affilée également, les six poèmes qui composent "Pluie oblique" de Fernando Pessoa Immédiatement et totalement... Ce fut le retour de Fernando Pessoa Alberto Caeiro à Fernando Pessoa à lui seul. Ou, mieux encore, ce fut la réaction de Fernando Pessoa contre son inexistence en Alberto Caeiro (Monteiro, 1985: 232-233).

Ces prémisses mentales s'accordent au principe théâtral de l'hétéronymie, qui est expliqué par Pessoa lui-même dans la "table bibliographique" de 1928 comme création d'un "drame en gens et non pas un drame en actes"12. C'est par allusion à Shakespeare et à la figure emblématique de Hamlet que le poète des Fictions de l'Interlude explique le jeu ambigu d'interrelations entre ses trois principaux hétéronymes:

Supposons qu'un suprême dépersonnalisé tel que Shakespeare, au lieu de créer le personnage Hamlet comme partie d'un drame, le créait en tant que simple personnage, sans drame. II aurait écrit, pour ainsi dire, le drame d'un seul personnage, un monologue prolongé et analytique (Lopes, 1985: 111-113).

\footnotetext{
10 Je renvoie sur ce point aux travaux de Dionísio Vila Maior, à commencer par sa lecture bakhtinienne de Pessoa (Vila Maior, 1994).

${ }^{11}$ Toutes les références en français à l'œuvre essayiste de Fernando Pessoa résultent de ma propre traduction du portugais et parfois de l'anglais.

${ }^{12}$ Cité d'après l'Archive digitale de l'auteur, projet conçu par la Maison Fernando Pessoa qui met à disponibilité de nombreux écrits pessoens à l'adresse:

http://multipessoa.net/typographia/labirinto/multipessoa-obra-publica-25.pdf. Consulté le 12 juillet 2011.
} 
Nous retrouvons ainsi au cœur de l'hétéronymie pessoenne l'idée mallarméenne de "drame avec soi”, que Pessoa a diffracté et irisé d'une gamme diverse de figures, celles-là mêmes qui évoluaient dans sa "scène spirituelle". Teresa Rita Lopes qui a escompté soixante-douze personnalités littéraires créées par Pessoa - en plus de l'auteur orthonyme ${ }^{13}$ - a remarqué le caractère multiple et multivoque du jeu dramatique pessoen: un "mouvement de flux et de reflux /qui/ ne se manifeste pas seulement à l'intérieur de chaque personnage, mais aussi dans leurs rapports entre eux" (Lopes, 1985: 452) et qui permet, en retour, "une action thérapeutique sur Pessoa" (ibid.: 450) lui-même.

Un peu à l'image de l'Aleph, le sujet peut voir tout ce qui existe dans son univers à la fois de son pôle central, orthonymique, que de ses angles corrélatifs, dans un mouvement dialectique de soi à l'autre. Le jeu de l'hétéronymie se réalise dans ce rapport entre le fini et l'infini, rapport 'libre' fait de reflets et d'échos, de duplications et de résonances. II se différencie bien de la logique arachnéenne et calculée de Mallarmé. "Je m'envole, c'est tout", écrit Pessoa: contrairement au poète d'Hérodiade accroché à son œuvre, telle une "araignée sacrée", au risque de s'y confondre, l'expérience poétique pessoenne n'est pas de l'ordre de l'identification mais de celui du déploiement hors de soi, mais médiatisé par soi ${ }^{14}$; une extériorisation à dimension dramatique par laquelle le sujet ne se pose pas comme matière ou comme identité d'un autre son égal (échappant ainsi à l'égotisme du pseudonyme) mais ouvre un jeu d'échanges et de variations, ouvert à toutes les réalisations. Adhérant totalement à cette ex-centricité relationnelle, le sujet forme avec 'ses autres' une unité. De façon ultime, la dialectique de l'identité et de l'altérité, et le jeu hétéronymique qui la soustend réalise l'espace nu et mobile d'un théâtre totalement virtuel, sans véritable support physique. C'est l'extension maximale de l'utopie théâtrale mallarméenne. A la différence de Mallarmé pourtant, le théâtre mental hétéronymique ne va pas de pair avec l'évidement de l'élément humain ou avec sa sublimation par le jeu abstrait des signes - comme l'allégorise bien la danseuse de Mallarmé qui 'n'est pas une femme qui danse' mais un "Signe" - il naît, au contraire, de l'éclosion et de l'incarnation dans un corps actant qui devient, lui-même, porteur d'une manière artistique.

La conception mentale des hétéronymes est aussi bien du domaine du délire, que traduit l'excès créatif, que de celui du désir qui trahit un manque. Le poète fait remonter le procédé "à ses six ans" et à l'apparition, en langue française et en mouvement, du "Chevalier de Pas" (Monteiro, 1985: 231). C'est ce compagnonnage singulièrement lié au monde de l'enfance et déjà paradoxal, de par la polysémie du mot pas, qui est à la base d'un phénomène de création unique qu'il va développer tout au long de sa vie: "c'est ainsi que j'ai

\footnotetext{
${ }^{13}$ Voir la section "Dramatis Personae" de son ouvrage Pessoa por conhecer (Lopes, 1990: 167-169).

${ }^{14}$ La mise en scène des Trois derniers de Fernando Pessoa d'Antonio Tabucchi par Denis Marleau (1997) livre de façon saisissante ce rapport co-créateur entre Pessoa et cinq de ses hétéronymes qui finissent par se superposer par le jeu des masques vidéoscéniques.
} 
trouvé et répandu, plusieurs amis qui n'ont jamais existé, mais que j'entends, je sens et je vois, aujourd'hui encore, à près de trente ans de distance. Je répète: j'entends, je sens, je vois... Et ils me manquent" (ibid.: 232). Le sentiment de perte y est premier, si l'on se souvient que l'ami imaginaire vient contrecarrer des absences bien concrètes dans la vie du jeune Fernando: la perte du père et en partie de la mère, remariée, l'abandon de la patrie, de la langue et civilisation d'origine pour l'Afrique du Sud (Durban) et la langue anglaise. L'hétéronymie est ici esquissée et le point sensible qui relie et inscrit, pour les restituer, ces présences alternatives, est le poème. Le poème est alors cet espace de co-naissance d'un autre, d'une personnalité autre, des sens autres et des mots autres. C'est en tout cas à cette connivence entre l'intellectuel et le sensoriel, entre l'affectivité et la raison, qu'il faut ramener la manière pessoenne, là où se rejoignent l'homme, le penseur et l'artiste. Car il ne s'agit pas d'un exercice de démence calculée, comme on pourrait le dire à propos du Coup de dés, mais d'un processus d'extériorisation du sensible qui confère à des valeurs telles que l'instabilité, la dispersion, la folie des enjeux esthétiques déterminants, inséparables d'une éthique: "não escrevo em português, escrevo eu mesmo" consigne Bernardo Soares dans Le Livre de l'intranquillité. L'œuvre de Fernando Pessoa est d'abord cela: volonté de dire, même si par voie - et par voix - négative ou paradoxale.

Le mot Pessoa, qui se traduit par 'personne' et, par extension, masque, recèle cette négativité qui est, paradoxalement, ouverture vers d'autres 'je', s'y coulant pour mieux se (re)connaître, dans l'effort de surmonter le conflit entre être et ne pas être, entre fiction et biographie, que la notion ambiguë de fingimento comme 'mentir plus vrai' contribue à renforcer. On se souvient du poème "Autopsychographie": "Le poète est un simulateur/ II simule si totalement/ qu'il arrive à simuler comme une douleur / La douleur qu'il ressent vraiment". Ce faisant, cette feintise est non seulement un concept unissant l'artiste et sa création - jusqu'à devenir geste de connaissance: "feindre c'est se connaître" - mais elle engage une dimension instinctive, voire 'sauvage' quand il s'agit de "tout sentir de toutes les manières possibles", comme le manifeste Álvaro de Campos, l'autre ingénieur visionnaire et poète sensationniste de Pessoa ${ }^{15}$. L'idée que la création procède d'une homologie entre sensation et conscience est bien exprimée dans la même lettre, où le poète pose la question de l'hétéronymie comme hypothèse pathologique - hystérie, aboulie, folie sont des mots qui reviennent. Ainsi Álvaro de Campos est-il "un complément véritable et hystérique" (ibid,: 234) de Fernando Pessoa et Bernardo Soares, le semi-hétéronyme, "c'est moi moins le raisonnement et l'affectivité" (ibid.,: 235). Outre sa désignation comme mouvement littéraire

\footnotetext{
${ }^{15}$ L'on peut rappeler ici l'important Ultimatum d'Álvaro de Campos (1917), où le poète dresse un portrait sévère et iconoclaste d'un "Portugal-centimes, reste de Monarchie pourrissant en République, extrême-onction-déshonneur de la Disgrâce", tout en proclamant l'avènement d'un "Sur-Homme /.../ le plus Complet".
} 
englobant $^{16}$ où il inscrit ses principaux hétéronymes - Campos, Reis, Caeiro - et lui-même comme "le plus purement intellectuel" (Pessoa, 2009: 216), le sensationnisme constitue en effet cet arrière-fond quasi cosmogonique qui permet d'envisager l'ex-centricité pessoenne comme processus qui relie polarisation et poétisation dans un mouvement dialectique.

Quoi qu'il en soit, c'est toujours en fonction du sentiment même de soi - désavouant la séparation entre raison et émotion - que le poète précise sa démarche:

en tant que poète, je sens; en tant que poète dramatique, je sens en me détachant de moi-même; en tant que dramaturge (sans poète), je convertis automatiquement ce que je sens en une expression étrangère à ce que j'ai senti, en construisant dans l'émotion une personne inexistante qui la sentirait vraiment et qui ainsi sentirait, dérivées de moi, d'autres émotions que moi, celui qui n'est que moi, j'ai oublié de sentir (Blanco (org.), 1986: 279).

De nombreux textes révèlent par ailleurs son vif intérêt pour les sciences ésotériques et l'astrologie - les cartes astrales des hétéronymes, établies par sa propre main, sont restées célèbres. Dans la lettre déjà citée, le poète conjecture trois chemins pour l'occulte: "le chemin magique /.../ le chemin mystique /et/ le chemin alchimique, le plus difficile et le plus parfait de tous, car il implique la transmutation de la personnalité elle-même" (Monteiro, 1985: 236).

Cette transmutation est donc à la fois propédeutique, visant à atteindre des plans inconnus - Pessoa va jusqu'à poser l'hypothèse de l'existence de mondes extra-terrestres qui jouxteraient "plusieurs échelles d'êtres" (237) - et constitutive puisqu'elle fait éclore des autres dotés d'une physionomie singulière ${ }^{17}$ et, au-delà, d'un ensemble de traits artistiques singuliers authentifiés par la signature hétéronymique. Caeiro, Reis, Campos ou Soares sont des écrivants à part entière, ils ont au regard de Pessoa orthonyme (selon sa propre désignation) une étrangeté ou une extravagance poétiques entièrement inscrites sous le signe de la fiction - qui peut atteindre les paroxysmes de la sensation 'incarnée', chez Campos, l'érudition la plus épurée chez Reis, ou le détachement primitif chez Caeiro. Insuffisante s'avère dans tous les cas la notion de style chez des auteurs dotés d'une biographie et d'une personnalité littéraire propres, et au-delà, d'un éthos particulier, qui implique une inscription dans la 'vie sociale'. Reis, qui est médecin, et Campos, qui est ingénieur, sont cosmopolites, alors que Caeiro a passé "toute sa vie à la campagne. II n'a pas exercé de profession et n'a pas, pour ainsi dire, reçu d'instruction".

\footnotetext{
16 “[...] qui accepte tous les systèmes et écoles d'art, extrayant de chacun d'eux la beauté et l'originalité qui lui est particulière" (Pessoa, 2009: 156).

17 Dans la même lettre Pessoa établit la biographie - "Je leur ai construit des âges et des vies" - et brosse les portraits - physiques et psychologiques - de ses trois principaux hétéronymes.
} 
Dans ce cadre, le concept de manière, au sens poétique et critique proposé par Gérard Dessons, peut permettre de cerner de plus près la singularité de Pessoa, inséparable de la pluralité qui se manifeste sous la main qui trace les mots. Reliant sens, histoire et sujet, ce concept prend en compte la dimension historicisée du jeu poétique et se distingue, à ce titre, de la notion de style, le mot manière impliquant, de fait, le sujet dans le poétique. Comme l'explique le critique dans L'art et la manière: "dans une œuvre picturale, musicale, ou littéraire, nous regardons, écoutons, lisons une manière singulière, c'est-à-dire non un objet du monde, mais une éthique du monde, une façon par la couleur, les sonorités, le langage, d'être dans le monde et au monde, une façon de faire du monde la tenue d'un sujet" (Dessons, 2004: 84).

Plus récemment, le concept s'est précisé par la complicité et la proximité étymologique qu'il noue avec la notion de folie, véhiculée par l'étymon 'mania', même si ce dernier a été retranché au glas du positivisme du XIXe siècle, au profit de manus et d'une définition 'stylistique' - mais finalement restrictive du phénomène de création artistique ${ }^{18}$. Le concept de manière folle, qui donne son titre au dernier essai de G. Dessons (2010), permet ainsi de penser la littérature comme un acte, et comme tel, engageant un sujet dont l'individuation ne s'éclaire qu'au contre-jour du mouvement interne de l'œuvre. Folle par essence, l'œuvre d'art tire sa force et sa signature de sa capacité singulière de participer à l'invention - langage, idée et forme à la fois - de mondes possibles. C'est pourquoi des notions denses, à teneur métaphysique, telles que l'indicible ou l'invisible ne sont pas pour autant indisibles (d'un point de vue linguistique et plus largement discursif): elles interpellent et magnifient d'autres manières de dire dont peut participer aussi bien la ponctuation - que l'on songe aux points de suspension, qui invitent au relais ou à l'usage du tiret suggérant un inter-dit (223) - que les formules oralisantes telles que le 'je ne sais quoi' (2010:105), révélant que la vie du langage dépasse l'esthétique ${ }^{19}$. La folie est incarnée dans l'œuvre, dont il faut percer l'écorce pour découvrir sa vivante nature, sa mouvance, et relier, par démarche compréhensive, deux pôles, distincts mais communicants: "la façon dont le langage signifie et /.../ la manière de lire, d'écouter et d'entendre", précise l'auteur (Dessons, 2010:118). La folie qui fait bruisser et bouger la matière - les mots - n'est compatible ni avec l'idée d'exclusivité ni avec celle d'exclusion ${ }^{20}$. Pour retrouver dans sa véritable portée l'indissociabilité fondamentale "entre une forme de langage et une forme de vie" (p. 194), suivant le principe fondamental défini par Henri Meschonnic, G. Dessons met l'accent sur les

\footnotetext{
${ }_{10}^{18}$ Voir la section "Manie et manière, une étrange histoire" (Dessons, 2010: 45-61).

${ }^{19}$ Même un déictique aussi (apparemment) infime - voire variante relâchée - que 'ça' (trans)porte "en même temps l'indexation et l'indétermination /.../ de là son efficience dans la représentation de l'inconscient chez Freud" (Dessons, 2010: 104).

${ }^{20}$ C'est pourquoi "l'œurre folle" peut irriter le critique lui-même. L'"excès rhétorique" (Dessons, 2010: 141), décelé dans les discours critiques concernant des auteurs extravagant les paradigmes (re)connus, tels que Nerval, Greco ou Aloysius Bertrand, est un beau symptôme d'une critique "qui s'affole" quand elle doit quitter les catégories génériques de la pensée.
} 
enjeux éthiques et politiques de certains choix poétiques, configurés en manière. Comment comprendre autrement "le populisme comme pensée du populaire" (p. 138) chez Céline? Envisagée du point de vue d'une continuité entre l'artiste et l'objet, une continuité qui est à la fois "trans-subjective" et "collective parce qu'elle circule" (p. 225), l'œuvre se dérobe aux codes et aux classifications a priori. Elle devient extravagante. C'est ce qui permet, comme le précise bien G. Dessons, "d'être non pas unique mais spécifique" (Dessons, 2010: 102). Articulée à celle de manière folle, la notion d'extravagance gagne alors un intérêt singulier pour appréhender l'hétéronymie par laquelle se réalise le double vœu de l'œuvre de Pessoa: la réciprocité entre écriture et psyché, qui met à l'épreuve le langage, et la transmutation aussi bien physique que mentale par la pensée et la sensation ouvrant et œuvrant de multiples configurations. Et, chemin faisant, l'individuel s'articule au collectif puisque c'est l'humain dans sa fragilité d'ego affectibus et dans sa complexité d'ego cogito qui se trouve traduit en œuvre d'art. En jeu hétéronymique interpelant un triple mouvement: rhétorique, stylistique et politique. La manière de Fernando Pessoa ne reste pas au niveau épidermique, elle est une manière intérieure, obsessionnelle. Elle est une manière folle.

\section{“N'est pas minuit qui veut” (René Char)}

Dans le sillage d'Hérodiade et du Faune composés à la même époque, Igitur se situe dan un rapport d'interférence, entre conte et drame. Énigmatique, tissé d'échos et d'emprunts au fantastique de Poe et de Shakespeare, Igitur pourrait avoir pour sous-titre "monologue avec Soi" - forme que Mallarmé avait célébrée dans ses pages critiques sur Hamlet - tant le combat du héros rejoint celui du célèbre personnage shakespearien: “l'antagonisme de rêve chez l'homme avec les fatalités à son existence départies par le malheur" (Mallarmé, 2003: 166).

Minuit sonne - Le Minuit où doivent être jetés les dés. Igitur descend les escaliers de l'esprit humain, va au fond des choses: en "absolu" qu'il est (Mallarmé, 2003: 474)

Après les tentatives dramatiques d'Hérodiade et du Faune en vue de la forme "non possible au théâtre mais exigeant le théâtre", Mallarmé approfondit son idée de théâtre mental qu'il esquisse dans lgitur aussi bien au niveau paratextuel comme au niveau textuel. Dès la dédicace, Mallarmé souligne bien la scénographie - par déplacement, par combinaison - destinée au lecteur - anticipant la "mise en scène spirituelle" énoncée dans la Préface du Coup de Dés: 
Ce conte s'adresse à l'Intelligence du lecteur qui met les choses en scène, elle-même (Mallarmé, 1998: 475)

Les indications théâtrales dans les ébauches du texte - comme dans le feuillet intitulé "Scène de Th. ancien Igitur" (ibid:: 477), les "arrières-textes" de Hamlet dès le sous-titre, el be non $^{21}$, l'architecture en quatre "morceaux" sont autant d'éléments qui inscrivent lgitur dans un horizon générique dramatique autant que narratif. Le drame psychologique de l'étrange personnage à l'encontre des - "Heures vides, purement négatives" (ibid.,: 485) et la pression du monde chaotique qui l'assaille, qui pèse sur l'existence de tout homme "avant que son Idée n'ait été complétée" (ibid:: 498) peut, par ailleurs, être perçu comme une allégorie du poète lui-même qui se dit à l'époque pris dans les "griffes du vieux Monstre", quoiqu'il laisse entendre une victoire imminente: "la conscience, excédée d'ombres, se réveille, lentement formant un homme nouveau"22.

"Dénué de toute présence", Igitur oscille entre la première et la troisième personne et se confond tantôt avec l'ombre, tantôt avec la Nuit ${ }^{23}$. Le "drame avec soi" se resserre jusqu'à la désubstanciation d'un personnage dont le nom est une conjonction, dans une 'langue morte', vidant le personnage comme matière et le faisant se développer comme Idée. Comme manière. Démarche - ou ressource - fondamentale qui, dans la logique de l'œuvre mallarméenne entérine le passage d'une logique de la représentation, fondée sur un paradigme mimétique, à une logique de la Fiction aux (en)jeux éminemment poétiques. Si penser c'est "secoue(r) les dés" ${ }^{24}$, le texte est alors cet espace où l'art d'écrire se déploie selon une logique composite plurielle - où se jouent "les combinaisons de l'infini vis-à-vis de l'absolu", comme le pose lgitur - comme on le retrouve dans Le Coup de dés. Tel est le geste fondamentalement poétique, réalisé par ce qui ressemble à une opération mathématique:

L'infini sort du hasard, que vous avez nié. Vous, mathématiciens expirâtes - moi projeté absolu. Devais finir en Infini. Simplement parole et geste.

C'est dans ce mouvement transportant un faire ou une manière poétique - dire et geste - qu'lgitur inscrit son tour de dés, installant la Folie au creux de l'œuvre et donnant

\footnotetext{
21 "Ne sois personne" formule que Jean-Pierre Richard considère comme une réponse négative et impérative "à la vieille interrogation shakespearienne" (Richard, 1961: 184).

22 Lettre du 19 février 1869 à Henri Cazalis (Mallarmé, 1998: 742) - c'est-à-dire quelques mois avant de commencer lgitur, dont les premières allusions apparaissent dans une lettre de juillet 1869).

${ }^{23}$ Comme le remarque Bertrand Marchal, "héros fictif a tous les points de vue, Igitur n'est en somme rien d'autre qu'un comédien, et son rapport à l'acte est proprement dit celui d'un acteur" (Mallarmé, 1998:1349).

${ }^{24}$ A la suite de nombreux commentateurs Bertrand Marchal rappelle l'ascendance d' lgitur sur le Coup de dés. Cf. sa "Notice d'Igitur" (Mallarmé, 1998: 1346- 1353).
} 
plus particulièrement au mystère de la vie intime - ce "labyrinthe de troubles et de griefs" que Mallarmé retrouve dans la figure de Hamlet - une scène de (ré)conciliation:

Ne sifflez pas parce que j'ai dit l'inanité de notre folie! silence, pas de cette démence que vous voulez montrer exprès. Et bien! il vous est si facile de retourner là-haut chercher le temps - et de devenir (Mallarmé, 1998: 481)

Ces deux axes - folie et connaissance - sont reliés par un même pacte ou, plus exactement, par un même geste conjurant "l'horreur /et/ l'accablante sensation de fini" (ibid: 499). Igitur ferme le livre, souffle la bougie "du souffle qui contenait le hasard" (ibid.) et se couche dans le tombeau de ses ancêtres, en croisant les bras, après avoir vidé la fiole /folie contenant "la goutte de néant qui manque à la mer" (ibid.: 478). Libéré de tout magistère, le voici définitivement enveloppé de beauté, développé dans le jeu libre autonome de la fiction. La dernière phrase est particulièrement significative: "le Néant parti, reste le château de la pureté" (ibid.). Le hasard est nié au moyen d'un Acte, qui est assomption volontaire de la "Folie", en assure la dimension créatrice et lui confère son statut poétique, comme le suggère le mot Idée, si conceptuel chez Mallarmé:

Mais l'Acte s'accomplit.

Alors son moi se manifeste par ceci qu'il reprend la folie: admet l'acte, et volontairement, reprend l'Idée, en tant qu'Idée. (ibid.,: 476)

ce que suggère encore le fragment suivant:

Nécessaire - extrait l'Idée. Folie utile. Un des actes de l'univers vient d'être commis là. (ibid.: 474)

La folie devient motrice - telle l'inquiétude de Pessoa -, permettant à la fois le détachement (au sens le plus littéral) d'une réalité contraignante et le déplacement avantageux en nouvel ordre cosmopoétique à partir duquel le monde se transmue en Fiction. C'est toute une expérience que ce mot résume, non seulement de la langue, mais aussi de son lieu de provenance, l'homme. Une telle conception comprend une portée éthique: cellelà même que Mallarmé ne cesse de réitérer dans ses définitions du livre comme "explication de l'homme".

En ouvrant à l'énergie pulsionnelle, au jeu de l'esprit, inventif, transgressif, Igitur consacre la folie contre le topos classique de la bouteille à la mer - la fiole de verre /qui/ renferme la substance du néant, la combinaison et la suggestion contre la référence et la 
nomination. Pour autant qu'il soit "absurde" son geste revêt un rôle catalyseur et analytique; il "réduit le hasard à l'infini" - l'image mathématique posant l'infini comme condition et comme méthode. C'est ici que "l'initiative [cédée] aux mots" fait corps avec l'invention générique. Dès lors, corollairement, est valorisée une démarche intellectualiste et une manière plus scientifique que philosophique. Igitur se constitue en 'œuvre folle', tout en faisant de la fiction son ressort intérieur - poétique et métapoétique. La page devient le seul lieu où “I'Infini est (enfin) fixé" - geste fou, geste vital, folie nécessaire:

...mais en même temps il peut dire que par le fait de cette folie, le hazard [sic] étant nié, cette folie était nécessaire. A quoi? (Nul le sait, il est désolé de l'humanité).

Tout ce qu'il en est, c'est que sa race a été pure: qu'elle a enlevé à l'Absolu sa pureté, pour l'être, et n'en laisser qu'une Idée elle-même aboutissant à la Nécessité: et que quant à l'Acte, il est parfaitement absurde sauf que mouvement (personnel) rendu à I'Infini: mais que l'Infini est enfin fixé. (ibid.: 477)

\section{"Ma pensée folle est succombée" (Pessoa, Faust, Tragédie subjective)}

Igitur et Faust, tragédie subjective se rejoignent, en dépit de tout ce qui peut les distinguer, dans l'idée commune de l'œuvre comme célébration du Mystère, qui se cherche non dans la transcendance mais dans l'immanence, dans les lettres. "Pour la Rose et le Lys le mystère d'un nom" écrit Mallarmé dans son "Toast Funèbre". Avec le Faust de Goethe à sa base, Pessoa met en œuvre une forme dramatique insolite, nourrie de mots plus que d'action, ouverte à la vie subjective. Mais le rapport que le personnage entretient avec la langue est de l'ordre de la contra-diction, en résonance avec les limites du sens et de la raison dans lesquels se meut et se confond Faust. Et vers lesquels il échoue: "O, Vie, tu t'en vas. Viennent des ombres. Aveugle. O Faust! (Il expire)" (Pessoa, 2008: 290).

Comme dans Igitur, la figure mise en scène est celle d'un héros dont l'origine est occultée, "reculé au-delà de toute vie possible" au sein d'un "drame" solitaire, né de la conscience d'exister. Le sous-titre "Tragédie subjective" choisi par Teresa Sobral Cunha pour sa reconstitution des fragments de Faust rend bien compte, comme le remarque Eduardo Lourenço dans la Préface à l'édition française, de la "théâtralisation conceptuelle" qui est en jeu dans un texte relevant d'une "poétique ontologiquement opposée à toute fermeture" (Pessoa, 2008: 9).

Les deux fragments liminaires révèlent d'emblée l'univers éclaté de ce texte qui n'obéit à aucun genre précis, dont la composition semble aimanter des éléments diffus d'un ailleurs où tout est semblable, où tout est différent, dans un miroitement sans passé ni futur. 
À la croisée entre poème dramatique et récit poétique, oscillant entre médiation et onirisme, il nous fait perdre nos repères pour nous convier à une aventure où tout est mystère - un des mots privilégiés de Faust, avec celui d'infini et d'horreur. Un "mystère" inaliénable et la certitude subreptice de l'échec - soit un fatum. L'espace du drame se (con)fond à celui de la pensée, alliant tragique et subjectivité - critique et clinique, pourrait-on dire avec Deleuze.

Après avoir renvoyé dos à dos le rêve et la science - quels palliatifs pour compenser l'illusion d'un monde impénétrable et hostile à ses élans de connaissance:

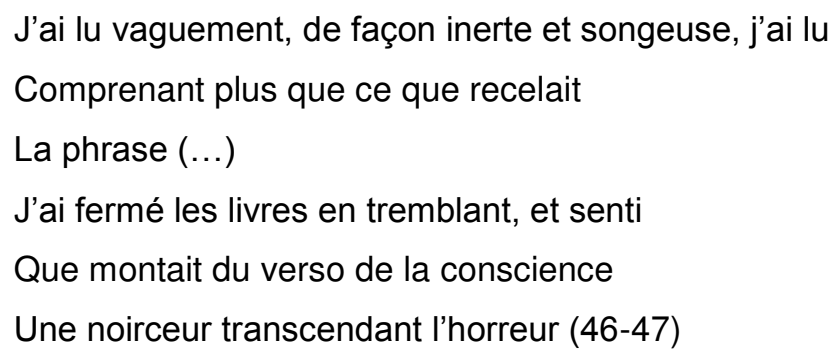

Faust, les yeux fermés, entreprend une longue descente vers le gouffre de la pensée jusqu'à discerner "un puits sans parois" (69), bientôt identifié aux ténèbres et à la mort autant d'éléments qui peuvent rappeler le huis-clos d'Igitur, dont on retrouve les motifs du miroir, du labyrinthe et de la nuit. Moins symétrique toutefois, c'est le tourbillon d'une pensée qui s'irise en écriture, en une folle ronde de mots, rendant douloureuse et dérisoire toute tentative d'unification:

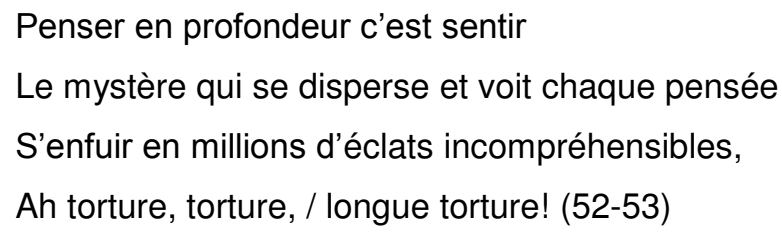

"Dans son laboratoire", le personnage ne dégage qu'un double abîme, qu'un double échec - de la religion comme de la connaissance. Égaré dans le labyrinthe du savoir - lieu de réflexes, d'affects et de percepts -, son discours épouse la dérive du sujet et sa découverte d'un monde toujours en attente de son contraire. Moins désincarnée que celle d'Igitur c'est une voix plus extravagante, plus extravasante, qui revient, perpétuellement, et accroît l'étonnement d'exister. L'obsession de Faust se "perd/ant/ à penser" (52) rejoint l'intranquillité foncière de celui dont le nom est Personne:

\section{L'INNOMABLE}

Dans mon abîme atroce

$$
\text { I...I }
$$


L'en-soi de l'Être n'est même pas le nom

De mon être indicible

Dans son Maelström muet

S'éteint comme un soupir

L'instable et vaste monde (99)

Épuisé d'intelligence, dépossédé de ses origines - "il est mort-né celui qui a voulu de moi" (148) -, son discours erre - "vaguement passe" - au rythme des images qui filent, irrepérables, en hallucinations de mots - "gouffres d'afflictions", "Gorgones et Démogorgon" (110-111) et qu'il fond en interminable spirale. Ses interpellations ne se posent toutefois que pour mieux révéler leur avers véritable: l'inintelligibilité du monde, que ni le "démontage impie" du logos, ni l'ironie méprisante ne parvient à conjurer:

Avec tant d'horribles possibilités

I...l

Non sans frémir, je l'ai abandonné

Au chaos de mon être, là où gisent ensemble

Les spectres noirs des réponses passagères

I...I

Et de terrifiants systèmes provisoires

I...I

Des Systèmes et des pensées

Qui ne font que rendre plus noir

Le pouvoir atroce qui les transcende

Tous, oui, tous, et infiniment

Ô horreur! Ô mystère! Ô existence!

De quel côté ne pas me tourner

Où vais-je ouvrir les yeux - les yeux de l'âme

Afin que le mystère ne me tourmente plus

Er que je n'aille pas vers lui en tremblant?

Et... pourquoi parler? Que dire? (105)

Tout le place inéluctablement en "erreur, errance éternelle" (64) dans un jeu aimant entre centre et absence, entre attraction et folie, vis-à-vis d'un univers où le transitoire se creuse jusqu'au paroxysme, où seul demeure le sentiment d'étrangeté et d'incompréhension (75) -, tel un serpent se mordant la queue: “... le fait de ne rien comprendre let de ne pas savoir pourquoi l'on ne comprend rien" (225). "J'ai dans le sang l'énigme de l'univers" (74) avoue-t-il enfin, dans une sorte de prise de conscience de l'écart suprême de vivre "ici DOULEUR, HORREUR au-delà" (82). Confondu à "la nuit froide", livré "au labyrinthe de 
/soi/même" (57), le monde apparaît comme un miroir multipliant en un jeu infini les apories, écartelant le sujet entre interrogation et abandon:

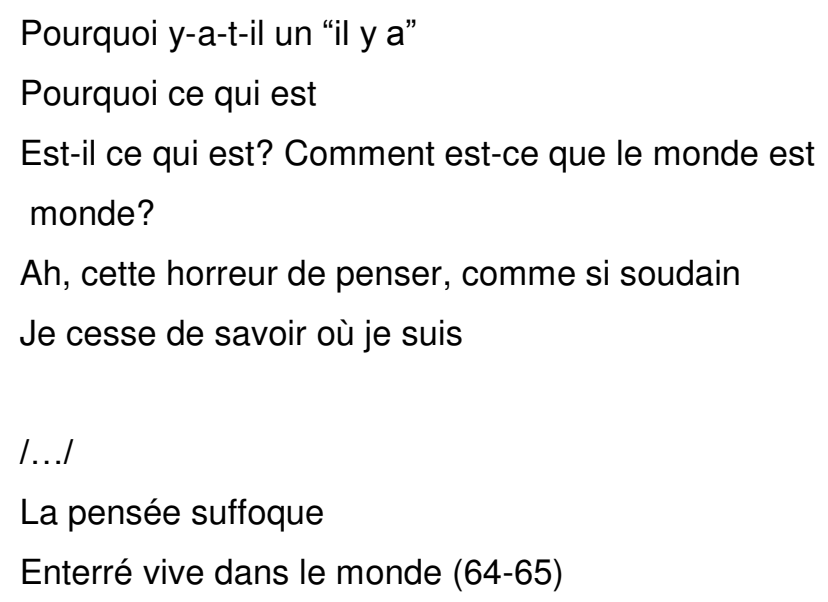

La désespérance fait crier à l' "agonie inconçue", à l' "agonie imaginante" métaphore-relais du poète lui-même et de son voyage au cœur de la vitalité comme de la déréliction du langage. Posant cette équation nihiliste: "Croire c'est mourir; penser c'est douter" (265) et dérogeant toute forme d'action - "Ah, I'horreur métaphysique de l'Action!" (248), la folie apparaît comme le seul moyen pour retrouver un sens; une logique de transgression qu'il associe aux "génies". Hors les frontières rationnelles, hors les "conventions /.../ de l'utile et de l'usuel", l'univers s'offre en domaine sans bornes. Le poète entremêle l'inconscience, le rêve et la folie dans un enchainement qui réunit l'appel de la connaissance, la démarche créatrice et la volonté d'y atteindre. C'est un des rares moments à orchestration polyphonique où résonnent, comme en oracle, Goethe, Shakespeare et "des Voix" - en écho à celles de Christ et de Buda:

\section{GOETHE}

(...)

Du fond de l'inconscience

De l'âme sobrement affolée

J'ai tire science et poésie

À foison

Merveilles de l'inconscient!

En rêve, j'ai créé d'autres rêves

Et le monde stupéfait

Sent comme est beau ce que je lui ai donné. 


\section{SHAKESPEARE \\ Et c'est la folie l'inspiration!}

DES VOIX

Seule est grande la folie!

Elle seule est bienheureuse! (89-90)

Sans doute y a-t-il ici un ressort de ce qui fait du geste artistique une "Folie utile" tel qu'il apparaît dans Igitur, et que la Folie entendue comme un acte créatif, délire lucide ou tout simplement Fiction, par laquelle s'opère chez Mallarmé comme chez Pessoa une victoire sur le "rien, qui est la vérité", une barrière à l'horreur, à l'inquiétude, et à l'irréductible mystère de notre condition. Ici Faust, comme auparavant Igitur et en arrière-plan Hamlet, œuvre à l'extériorisation de la tragédie intime et ce geste orienté vers l'à-venir, par ce qu'il opère et sollicite de renouveau, configure la manière de l'artiste comme promesse de sens et d'humanité, contre le hasard. Où vient s'inscrire Mallarmé: “Une ordonnance du livre de vers poind innée ou partout, élimine le hasard" (Mallarmé, 2003: 211).

Du lancement de dés d'Igitur, "fin de parole et geste unis" dans la tentative arachnéenne de fixer l'absolu, à la frénésie intérieure du Faust écartelé, selon les termes de Pessoa lui-même, "entre l'Intelligence et la vie", le combat donne une résonance particulière à la "fascination parente de l'angoisse" incarnée par Hamlet "seigneur latent qui ne peut devenir" et "ombre juvénile de tous" (Mallarmé, 2003: 167). De telles apories se coulent dans des formes extravagantes traversées de voix qui installent la vie de l'esprit et la folie comme un attribut avantageux. Les manières déployées s'avèrent ainsi un mouvement de mise en crise de la rationalité, par lequel l'œuvre semble se dérober au démon des frontières et des systèmes pour dire, de l'intérieur, les soupçons qui la submergent et les (res)sources subjectives qui la meuvent - ces mécanismes inconscients qui restent, aujourd'hui, une véritable boite de pandore pour les sciences humaines, comme pour les sciences dites "dures"25.

\footnotetext{
${ }^{25}$ Je pense notamment aux travaux d'António Damásio, dans le domaine des neurosciences, prônant une indissociable complémentarité entre corps et cerveau, entre raison et émotion ou, si l'on veut, entre sens et science. Voir par exemple L'Erreur de Descartes (Odile Jacob, 1995) Le Sentiment même de soi: corps, émotions, conscience (Odile Jacob, 1999), ou L'Autre moi-même. La construction du cerveau conscient (Odile Jacob, 2010). Dans cette dernière, Damásio fait observer, d'après plusieurs exemples repris de Crime et Châtiment, combien Dostoïevski est allé loin dans le champ de l'observation de la vie du cerveau, aspect qu'il souligne une entrevue récente au Jornal de Letras se référant aussi à Shakespeare et à Pessoa (qu'il cite en exergue à son dernier livre), Fitzgerald ou Faulkner. II y développe l'idée selon laquelle le fonctionnement du cerveau tient de l'organisation et de la dynamique d'une scène de théâtre. "Le théâtre /est/ une métaphore très puissante pour évoquer ce qui se passe dans le cerveau", affirme-t-il. Voir Jornal de Letras, 5 a 18 de Outubro de 2011, p. 29.
} 
La 'manière folle' d'Igitur et de Faust s'inscrit, au final, dans la continuité du soupçon du genre théâtral et plus spécifiquement tragique auquel, un siècle plus tôt, Victor Hugo posait son bonnet rouge par l'entremise d'Hernani et de son personnage éponyme. "Acte /.../ de Folie utile", chez l'un, conscience douloureuse de perpétuellement errer dans "l'éternelle erreur, dans l'éternel voyage" chez l'autre, le façonnement du jeu poétique de Mallarmé et de Pessoa passe par une théâtralité paradoxale, qui s'énonce comme théâtre mental, $a$ contrario sensu de la dramaturgie classique, chez Mallarmé, et qui acquiert toute son excentricité dans le jeu hétéronymique pessoen.

Toujours en devenir, le poème se déploie en geste continuel, dessinant ce mouvement perpétuel des signes qui participe à l'historicité. II s'offre en variation de l'aleph de Borgès - il est symbole de survivance, plus que de simple vie. 


\section{Bibliographie}

ARTAUd, Antonin $(1925,2004)$ Position de la Chair, in CEuvres, Ed Évelyne Grossman, Paris: QuartoGallimard.

BLANCO, José (org.) (1986). Pessoa en personne, Lettres et documents choisis par José Blanco, Paris: La différence.

BORGES, José Luiz (1967). "La quête d'Averroës", in L'Aleph, trad. par R. Caillois, Paris: Gallimard.

CABRAL, Maria de Jesus (à paraître) '“En spectacle (in)interrompu': Pessoa, Maeterlinck, Beckett selon Denis Marleau", in Cadernos de Literatura Comparada, Transbordamentos infinitos: a dramaturgia contemporânea, org. Alexandra Moreira da Silva et al., Porto: Edições Afrontamento.

CASA FERNANDO PESSOA, Biblioteca digital de Fernando Pessoa, Lisboa.

$<U R L:$ http://multipessoa.net/typographia/labirinto/multipessoa-obra-publica-25.pdf> [consulté le 12 juillet 2011].

DESSONS, Gérard (2010). La manière folle: essai sur la manie littéraire et artistique, Paris: Manucius. (2004). L'art et la manière - art, littérature, langage, Paris: Honoré Champion.

Hugo, Victor (s/d). Cromwell, in CEuvres complètes de Victor Hugo, Paris: Nelson Editeurs.

GAFFIOT, Félix (1934). Dictionnaire illustré latin français, Paris: Hachette.

LATOUR, Geneviève (org) (2000) Les extravagants du théâtre: de la Belle Epoque à la drôle de guerre. Catalogue de l'exposition présentée à la Bibliothèque historique de la ville de Paris, Paris: Paris bibliothèques.

LOPES, Teresa Rita (1985). Fernando Pessoa et le Drame Symboliste: héritage et création, $2^{2}$ ed., Paris: Fundação Calouste Gulbenkian.

(1991). Pessoa por conhecer, Lisboa: Editorial Estampa.

MALLARMÉ, Stéphane $(1998,2003)$ CEuvres complètes, édition présentée, établie et annotée par Bertrand Marchal, Paris: Gallimard, Bibliothèque de la Pléiade, t. I et II.

(1995). Correspondance, Lettres sur la poésie éd. Bertrand Marchal (Préface d'Yves Bonnefoy), Paris: Gallimard, coll. "Folio- Classique" ( $\left.n^{\circ} 2678\right)$.

PESSOA, Fernando (2009). Sensacionismo e Outros Ismos. Edição de Jerónimo Pizarro. Lisboa: Imprensa Nacional-Casa da Moeda. Edição Crítica de Fernando Pessoa, Série Maior, vol. X.

(2008). O Livro do Desassossego, Vicente Guedes. Bernardo Soares, edição de Teresa Sobral Cunha, Lisboa: Relógio d’Água.

(2006). Escritos sobre Génio e Loucura, edição de Jerónimo Pizarro, Lisboa: Imprensa Nacional - Casa de Moeda.

(2001). CEuvres poétiques complètes, Paris: Gallimard, Bibliothèque de la Pléiade.

(1985). Fausto Tragédia subjectiva, texto estabelecido por Teresa Sobral Cunha, Lisboa:

Editorial Presença.

(1966). Páginas Íntimas e de Auto-Interpretação, textos estabelecidos e prefaciados por G.R. Lind e Jacinto do Prado Coelho, Lisboa: Ática Editores.

VILA MAIOR, Dionísio (1994). Fernando Pessoa - Heteronímia e Dialogismo, Coimbra: Almedina.

Szond, Peter, (1983). Théorie du Drame moderne, 1880-1950, trad. Patrick Pavis Lausanne, l'Age d'Homme. 